\title{
Minimal Hepatic Encephalopathy and Neuroinflammation
}

\author{
Marcotegui Ariel R ${ }^{1 *}$, Cantillo Germán ${ }^{2}$, Souto Pablo A ${ }^{1}$, Grimaldi Santiago ${ }^{1}$, Orbea Li-sandro ${ }^{1}$, Eizayaga Francisco ${ }^{1}$, \\ Romay Salvador ${ }^{1}$, Lago Nestor ${ }^{1}$ and Perazzo Juan $\mathrm{C}^{1}$
}

${ }^{1}$ Department of Applied and Experimental Pathology, University of Buenos Aires, Argentina

${ }^{2}$ Universidad Pontificia Bolivariana, Colombia

*Corresponding author: Marcotegui Ariel R, Laboratory of Hepatic Encephalopathy and Portal Hypertension, Center of Applied and Experimental Pathology, University of Buenos Aires, Buenos Aires, Argentina

Submission: 監August 27, 2018; Published: 監 October 25, 2018

\begin{abstract}
Hepatic Encephalopathy (HE) is one of the major complications of acute or chronic liver failure. Its neurological and mental symptoms range from subtle behavioral abnormalities to deep coma and death. In addition, a subclinical HE state, named minimal HE (mHE) is a very frequent misdiagnosed complication. Although the etiology of hepatic encephalopathy is not fully elucidated, hyperammonemia plays a key role in its development. In liver diseases, its reduced detoxifying capability results in increased blood and brain ammonia concentrations. Under this condition, ammonia is neurotoxic to both neuronal and astrocyte cells in the CNS. Hyperammonemia is also an essential promotor of neuroinflammation increasing proinflammatory cytokines such as IL-1 $\beta$ and IL-6. It is known that even without morphological liver alterations, there are changes in the pro-inflammatory cytokines release and increase in the BBB permeability among other alterations. The aim of this review is to approach the neuroinflammatory aspects of minimal hepatic encephalopathy, where there are no clear manifestations of the disease.
\end{abstract}

Keywords: Minimal hepatic encephalopathy; Hyperammonemia; Neuroinflammation; Liver disease; Microglia; Astrocyte; AM404

Abbreviations: HE: Hepatic Encephalopathy; mHE: Minimal HE; CLF: Chronic Liver Failure; AASLD: American Association for the Study of Liver Diseases; EASL: European Association for the Study of the Liver; CNS: Central Nervous System; ROS: Reactive Oxygen Species; BBB: Blood-Brain Barrier; LPS: Lipopolysaccharide; GS: Glutamine Synthetase; MMP: Mitochondrial Membrane Potential; NO: Nitric Oxide; AQP: Aquaporins; NKCC1: Na-K-Cl Cotransporter-1

\section{Introduction}

HE: Hepatic Encephalopathy (HE), one of the major complications of acute or chronic liver failure (CLF) [1], is defined as the spectrum of neuropsychiatric abnormalities seen in patients with liver dysfunctions and/or portal systemic shunting after exclusion of other known brain diseases [2]. The neurological and mental symptoms are potentially reversible and can range from subtle behavioral abnormalities to deep coma and death [3]. Recently the American Association for the Study of Liver Diseases (AASLD) and European Association for the Study of the Liver (EASL) proposed a new classification of HE according to the following four factors:

1. The underlying disease,

2. The severity of manifestations,

3. The time course and

4. The existence of precipitating factors [4]. Furthermore, it is important to point out that mHE, a subclinical HE state, $\mathrm{mHE}$ [5] is a very frequent misdiagnosed complication [1].

HE displays hyperammonemia due to the impossibility of the non-functional or by-passed liver to metabolize it [6,7]. Under physiological conditions, more than $98 \%$ of ammonia is present in its ionic form $\left(\mathrm{NH}_{4}^{+}\right)$. Hence, while $\mathrm{NH}_{3}$, non-ionic ammonia, is freely diffusible across cellular and sub-cellular membranes, $\mathrm{NH}_{4}^{+}$ requires an ion channel or carrier transport system. In different models displaying hyperammonemia, severe changes in the central nervous system (CNS) were reported. Among them, mitochondrial dysfunction of the hippocampus [8], astrogliosis with increased reactive oxygen species (ROS) and the production of proinflammatory cytokines [7], which lead to mHE [9]. Therefore, in this context, HE is considered an indicator of poor prognosis in liver diseases [10].

\section{Pathophysiology}

Although the pathophysiology of HE is not completely elucidated, ammonia plays a key role [11]. Hyperammonaemia alters the integrity of the blood-brain barrier (BBB) in rats, increasing the influx of toxic substances into the brain [12]. Norenberg et al. [13] described, in an in vitro model, mitochondrial dysfunction oxidative and nitrosative stress as the main source of free radicals in the CNS under hyperammonaemia conditions [13].

The bacterial lipopolysaccharide (LPS), and the ammonia produced by the intestinal bacteria arrive through the portal vein, and 
are eliminated, respectively, by the reticuloendothelial system or metabolized in the liver. Therefore, the intestinal microbiota is an important factor in the development of HE. Endotoxemia can occur due to:

\section{An infection [14],}

2. Increased intestinal permeability [15] or

3. Deterioration of the hepatic reticuloendothelial system. Liver diseases reduce the detoxifying capacity of this organ, increasing the ammonia blood and brain concentrations. In addition, when these mechanisms are altered, the bacterial antigens, such as LPS, reach the general circulation activating the immune response [16]. LPS stimulates the microglial production of iNOS and NO [17], which contributes to the neuroinflammatory state in HE.

Hyperammonemia is neurotoxic for both neurons and astrocytes. The brain metabolizes ammonia mainly to glutamine by the enzyme glutamine synthetase (GS), which is located almost exclusively in astrocytes [18]. Once the glutamine is synthesized, it is transported to the mitochondria and then to the mitochondrial matrix where it is hydrolyzed into ammonia and glutamate, generating ROS [11,19] lipid peroxidation and depletion of glutathione and ATP. This condition decreases the mitochondrial membrane potential (MMP) that induces mitochondrial swelling [20]. In vitro studies indicated that the p47-NADPH oxidase pathway is involved in this process [21]. The production of free radicals increases due to lipid peroxidation, to a reduction in the activity of glutathione peroxidase and superoxide dismutase. In addition, the activation of glutamate receptors (NMDA) induces the nitration of proteins such as GS, with the consequent increase in the concentrations of brain ammonia, deterioration of brain energy metabolism, HE, cerebral edema [22], increase in nitric oxide (NO) and oxidative stress damage [23].

At this point, it could be asked if hyperammonemia induces damage in the CNS per se, through a neuro-inflammatory condition or both. Could mHE also be considered as the initial step in neuro-pathophysiological changes in ALF or CLF?

Hyperammonemia is also an essential promotor of neuroinflammation through the induction of astrocytosis and the activation of microglia, releasing proinflammatory cytokines such as IL-1 $\beta$ and IL-6, associated with altered membrane expression of AMPA, NMDA and GABA receptors [23]. The ROS produced by the pro-inflammatory state, as in the presence of LPS antigens, induce an increase in the permeability of the internal mitochondrial membrane that leads to the functional alteration of the respiratory chain. It was demonstrated in vitro that astrocytes treated simultaneously with ammonia and proinflammatory cytokines increased mitochondrial permeability compared to the administration of cytokines alone. In addition, antioxidants blocked this additive effect, indicating that oxidative stress represents an important mechanism in mitochondrial deterioration [24].

There is a consensus that the activation of microglia must be present in neuroinflammation. Häussinger et al. demonstrated that ammonia directly activates microglia in animal models; however, they found no increase in the induction of iNOS, COX-2 and in the release of prostaglandins, glutamate, proinflammatory cytokines and monocyte chemoattractant protein 1 (MCP-1). In addition, they also found microglial activation in the cerebral cortex of rats exposed to ammonia and post-mortem brain tissue from patients with liver cirrhosis and HE, but not from patients who had cirrhosis without HE [25]. In addition, it was demonstrated that in advanced stages of acute liver failure (ALF) with HE, the proinflammatory cytokines TNF- $\alpha$, IL-1 $\beta$ and IL- 6 were produced by astrocytes [26].

Astrocytes are the cells most importantly involved in the pathogenesis of mHE/ HE. Together with microglia, they release proinflammatory cytokines such as IL-1 $\beta$, which compromise the integrity of the BBB through the cyclooxygenase pathway [27]. In addition, IL-1 $\beta$ induces, through TNF- $\alpha$ and endothelin-1, increased permeability of BBB with deterioration of its function [28]. It was also shown, in cultured astrocytes, that IFN- $\gamma$ positively regulates the expression of iNOS [29]. In addition to astrocytes and microglia, immune cells in the periphery can produce proinflammatory cytokines because of CNS inflammation. These cytokines exert their effect on the brain by three different routes:

1. Peripheral tissues, which are innervated by the peripheral and autonomic nervous system and can send direct signals to the brain through the peripheral nerves;

2. Brain vasculature that can transmit signals through secondary messengers, such as NO or prostanoids (produced in response to cytokines); and

3. Cytokines can act directly at the brain parenchyma level after crossing the BBB or after entering areas of the brain that lack BBB [30]. Inflammatory cytokines can also inhibit the uptake of glutamate by astrocytes through a mechanism involving NO [31] and can modulate the diffusion of ammonia [32].

Proinflammatory cytokines such as TNF- $\alpha$, IL-18, IL-6, endotoxins and ammonia were significantly elevated in the plasma of cirrhotic patients with mHE compared with both cirrhotic patients without mHE and healthy controls [33]. Montoliu et al. [34] showed that serum levels of IL- 6 and IL-18 could discriminate cirrhotic patients with and without mHE [34]. In addition, TNF- $\alpha$, IL-1 and IL-6 induce the production of $\mathrm{NO}$ and prostanoids by the BBB endothelium. Through this mechanism, the inflammatory effect in the CNS occurs without histological damage of the BBB.

On the other hand, the alteration of the BBB tight junction was registered in the BBB Zonula Occludens-1 (ZO-1) area in the capillaries of the hippocampus in a mHE model, also displaying extracellular and astrocytic edema [35]. In addition, endothelial cells show mitochondrial damage and increased NOS activity [8]. Moreover, it has been suggested that the BBB impairment induces microglial reactions, such as the release of cytokines or the modulation of immunomolecules [36]. Rodrigo $\mathrm{R}$ et al. [37] demonstrated that chronic hyperammonemia is sufficient to induce microglial activation and HE neuroinflammation [37]. In addition, admin-istration of intra- 
peritoneal LPS in rats with bile duct ligation exacerbated CNS cytotoxic edema and even reached coma [38].

A major change in mHE is vasogenic and cytogenic edema of astrocytes, as observed in both ALF and CLF [39]. Changes in ion channel control affect cellular homeostasis and may eventually promote vasogenic edema. One of the most important ion exchangers is the Na-K-Cl cotransporter-1 (NKCC1) which plays an important role in the inflammation of astrocytes due to hyperammonemia [40]. In addition, there are channels such as aquaporins (AQP) that also play a key role in maintaining the BBB integrity. In CNS, AQP4 and AQP-9 are mainly found in astrocytes, while AQP-1 is in the choroid plexus. AQP-4 seems to be one of the most important isoforms in the development of brain edema observed in both ALF and CLF. Furthermore, this aquaporin has been related to the apoptotic pathway through the activation of caspase 3 and 8 and the stimulation of TNF- $\alpha$ and IL-1 $\beta$ [41].

In chronic experimental hyperammonemia with $\mathrm{mHE}$, once the portal pressure returns to normal, the integrity of the BBB is restored [42]. In animal models of acute-on-chronic liver failure, behavioral and BBB alterations were observed [43]. Toll-like receptor-4 (TLR4) also plays an important role in the development of neuroinflammation. In vitro experiments showed that the increased expression of TLR4 due to an ammonia stimulation contributed to the inflammation of astrocytes and cerebral edema [44]

Recently, a paracetamol metabolite, AM404 (N- (4-Hydroxyphenyl) -eicosa-5,8,11,14-tetraenamide) has demonstrated the ability to reduce the inflammatory mediators of the arachidonic acid pathway in microglial and organotypic cultures [45]. AM404 decreased the production of IL-1 and IL-6 in LPS induced neuroinflammation in rats [46]. In a neuropathic pain rat model, AM404 prevented overproduction of nitric oxide (NO) and $\mathrm{TNF} \alpha$, as well as increased production of IL-10 [47]. In addition, this metabolite of paracetamol inhibited the activity of the COX-1 enzyme and COX-2 isolated ex vitro and the formation of PGE2 in macrophages RAW 264.7 [48]. AM404 has also shown to be a potent agonist of the transient receptor potential vanilloid type 1 (TRPV1) and low affinity ligand of the cannabinoid receptor type 1 (CB1) [48]. Furthermore, it has been found the presence of this metabolite in the cerebrospinal fluid of patients after the administration of paracetamol [49].

\section{Conclusion}

This review is an attempt to address neuroinflammation and mHE. It is important to implement an accurate diagnosis when the clinical manifestations of mHE are still unclear. The question about whether hyperammonemia and/ or neuroinflammation start alone or associated the CNS alterations cannot be answered. It is known that even without hepatic histopathological alterations, type BHE, shows changes in the release of proinflammatory cytokines and increased permeability of BHE among others.

The astrocytes were designated as the first affected cells; however, more recently, in vitro studies showed that microglial activation and release of proinflammatory cytokines were early events. In addition, our laboratory observed neuronal morpho-functional dam-age in hyperammonemic conditions in organotypic cultures (unpublished results). The mechanism by which paracetamol damages the CNS yet is not fully understood, although AM404 could be considered as a possible candidate to reduce neuroinflammation and the oxidative stress pathways of mHE.

Neuroinflammation is a basic problem in the development of HD, but when does it really start? What is the starting point where the systemic inflammation and/ or the hyperammonemic condition activate the microglia and induce vasogenic edema in the CNS due to the alteration of the BBB? All these questions remain unanswered, so it is necessary to work more to diagnose this highly prevalent pathology earlier and develop a more specific treatment (Figure 1).

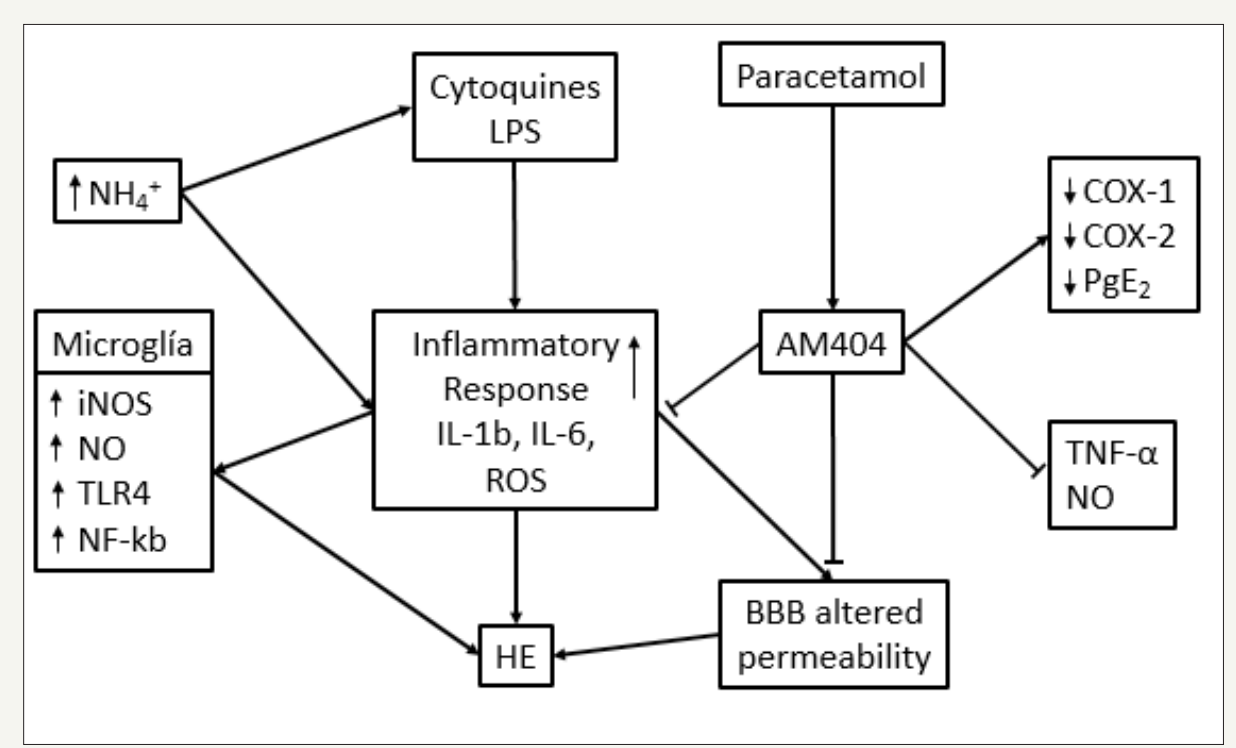

Figure 1: Neuroinflammation and HE. Interaction between ammonia (NH4+), lipopolisacharide (LPS), and citoquines that lead to hepatic encephalopathy and the AM404 inhibitory role. (Modified from [24]) 


\section{References}

1. Quero JC, Hartmann IJ, Meulstee J, Hop WC, Schalm SW (1996) The diagnosis of subclinical hepatic encephalopathy in patients with cirrhosis using neuropsychological tests and automated electroencephalogram analysis. Hepatology 24(3): 556-560.

2. Butterworth RF, Vaquero J (2009) Hepatic Encephalopathy. In: Arias MI, et al. (Eds.), The Liver: Biology and Pathobiology. ( ${ }^{\text {th }}$ Edn), John Wiley \& Sons Ltd, New Jersey, United States, pp. 1216.

3. Bosoi CR, Rose CF (2009) Identifying the direct effects of ammonia on the brain. Metab Brain Dis 24(1): 95-102.

4. Vilstrup H, Amodio P, Bajaj J, Cordoba J, Ferenci P, et al. (2014) Hepatic encephalopathy in chronic liver disease: 2014 Practice Guideline by the American Association for the Study of Liver Diseases and the European Association for the Study of the Liver. Hepatology 60(2): 715-735

5. Butterworth RF, Norenberg, Felipo V, Ferenci P, Albrecht J, et al. (2009) Experimental models of hepatic encephalopathy: ISHEN guidelines. Liver Int 29(6): 783-788.

6. Parekh PJ, Balart LA (2015) Ammonia and Its Role in the Pathogenesis of Hepatic Encephalopathy. Clinics in Liver Disease 19(3): 529-537.

7. Suraweera D, Sundaram V, Saab S (2016) Evaluation and Management of Hepatic Encephalopathy: Current Status and Future Directions. Gut and Liver 10(4): 509-519.

8. Lores-Arnaiz S, Perazzo JC, Prestifilippo JP, Lago N, D’Amico G, et al. (2005) Hippocampal mitochondrial dysfunction with decreased mtNOS activity in prehepatic portal hypertensive rats. Neurochem Int 47(5): 362-368.

9. Tallis S, Laura R. Caltana Pablo A. Souto Amalia E. Delfante Néstor R Lago, et al. (2014) Changes in CNS cells in Hyperammonemic portal hypertensive rats. Journal of Neurochemistry 128(3): 431-444.

10. Ginés P, Quintero E, Arroyo V, Terés J, Bruguera M, et al. (1987) Compensated cirrhosis: natural history and prognostic factors. Hepatology 7(1): 122-128.

11. Albrecht J, Norenberg (2006) Glutamine: A Trojan horse in ammonia neurotoxicity. Hepatology 44(4): 788-794.

12. Rose C, Butterworth RF, Zayed J, Normandin L, Todd K, et al. (1999) Manganese deposition in basal ganglia structures results from both portal-systemic shunting and liver dysfunction. Gastroenterology 117(3): 640-704

13. Norenberg, Rama Rao KV, Jayakumar AR (2004) Ammonia neurotoxicity and the mitochondrial permeability transition. J Bioenerg Biomembr 36(4): 303-307.

14. Hung TH, Lay CJ, Chang CM, Tsai JJ, Tsai CC, et al. (2013) The effect of infections on the mortality of cirrhotic patients with hepatic encephalopathy. Epidemiol Infect 141(12): 2671-2678.

15. Hakansson A, Molin G (2011) Gut microbiota and inflammation. Nutrients 3(6): 637-682.

16. Most D, Ferguson L, Harris RA (2014) Molecular basis of alcoholism. Handb Clin Neurol 125: 89-111.

17. Fiebich BL, Butcher RD, Gebicke-Haerter PJ (1998) Protein kinase C-mediated regulation of inducible nitric oxide synthase expression in cultured microglial cells. J Neuroimmunol 92(1-2): 170-178.

18. Cooper AJ, Plum F (1987) Biochemistry and physiology of brain ammonia. Physiological reviews 67(2): 440-519.

19. Rama Rao KV, Norenberg (2014) Glutamine in the pathogenesis of hepatic encephalopathy: The Trojan horse hypothesis revisited. Neurochem Res 39(3): 593-598.
20. Niknahad H, Akram J, Reza H, Mahdi Z, Mohammad Mehdi Ommati (2017) Ammonia-induced mitochondrial dysfunction and energy metabolism disturbances in isolated brain and liver mitochondria, and the effect of taurine administration: relevance to hepatic encephalopathy treatment. Clinical and Experimental Hepatology 3(3): 141-151.

21. Reinehr R, Boris Görg, Stephan Becker, Natalia Qvartskhava, Hans J Bidmon, et al. (2007) Hypoosmotic swelling and ammonia increase oxidative stress by NADPH oxidase in cultured astrocytes and vital brain slices. Glia 55(7): 758-771.

22. Butterworth RF (2016) Pathogenesis of hepatic encephalopathy in cirrhosis: the concept of synergism revisited. Metabolic Brain Disease 31(6): 1211-1215.

23. Hernández-Rabaza V, Cabrera-Pastor A, Taoro-González L, Malaguarnera M, Agustí A, et al. (2016) Hyperammonemia induces glial activation, neuroinflammation and alters neurotransmitter receptors in hippocampus, impairing spatial learning: reversal by sulforaphane. J Neuroinflammation 13(1): 41.

24. Jayakumar AR, Rama Rao KV, Norenberg MD (2015) Neuroinflammation in hepatic encephalopathy: Mechanistic aspects. Journal of Clinical and Experimental Hepatology 5(S1): S21-S28.

25. Zemtsova I, Görg B, Keitel V, Bidmon HJ, Schrör K, et al. (2011) Microglia activation in hepatic encephalopathy in rats and humans. Hepatology 54(1): 204-215

26. Wright GA, Sharifi Y, Newman TA, Davies N, Vairappan B, et al. (2014) Characterisation of temporal microglia and astrocyte immune responses in bile duct-ligated rat models of cirrhosis. Liver Int 34(8): 1184-1191.

27. de Vries HE, Blom-Roosemalen MC, van Oosten M, de Boer AG, van Berkel T], et al. (1996) The influence of cytokines on the integrity of the blood-brain barrier in vitro. J Neuroimmunol 64(1): 37-43.

28. Didier N, Romero IA, Créminon C, Wijkhuisen A, Grassi J, et al. (2003) Secretion of interleukin-1beta by astrocytes mediates endothelin-1 and tumour necrosis factor-alpha effects on human brain microvascular endothelial cell permeability. J Neurochem 86(1): 246-254.

29. Falsig J, Latta M, Leist M (2004) Defined inflammatory states in astrocyte cultures: correlation with susceptibility towards CD95-driven apoptosis. J Neurochem 88(1): 181-193.

30. Licinio J, Wong ML (1997) Pathways and mechanisms for cytokine signaling of the central nervous system. Journal of Clinical Investigation 100(12): 2941-2947.

31. Hu S, Sheng WS, Ehrlich LC, Peterson PK, Chao CC (2000) Cytokine effects on glutamate uptake by human astrocytes. Neuroimmunomodulation 7(3): 153-159.

32. Duchini A, Govindarajan S, Santucci M, Zampi G, Hofman FM (1996) Effects of tumor necrosis factor-alpha and interleukin- 6 on fluid-phase permeability and ammonia diffusion in CNS-derived endothelial cells. J Investig Med 44(8): 474-482.

33. Jain L, Sharma BC, Sharma P, Srivastava S, Agrawal A, et al. (2012) Serum endotoxin and inflammatory mediators in patients with cirrhosis and hepatic encephalopathy. Dig Liver Dis 44(12): 1027-1031.

34. Montoliu C, Piedrafita B, Serra MA, del Olmo JA, Urios A (2009) IL-6 and IL-18 in blood may discriminate cirrhotic patients with and without minimal hepatic encephalopathy. J Clin Gastroenterol 43(3): 272-279.

35. Perazzo JC, Tallis S, Delfante A, Souto PA, Lemberg A, et al. (2012) Hepatic encephalopathy: An approach to its multiple pathophysiological features. World J Hepatol 4(3): 50-65

36. Jensen MB, Finsen B, Zimmer J (1997) Morphological and immunophenotypic microglial changes in the denervated fascia dentata of adult rats: correlation with blood-brain barrier damage and astroglial reactions. Exp Neurol 143(1): 103-116. 
37. Rodrigo R, Cauli O, Gomez-Pinedo U, Agusti A, Hernandez-Rabaza V, et al. (2010) Hyperammonemia induces neuroinflammation that contributes to cognitive impairment in rats with hepatic encephalopathy. Gastroenterology 139(2): 675-684.

38. Wright G, Davies NA, Shawcross DL, Hodges SJ, Zwingmann C, et al (2007) Endotoxemia produces coma and brain swelling in bile duct ligated rats. Hepatology 45(6): 1517-1526.

39. Blei AT (2005) The pathophysiology of brain edema in acute liver failure. Neurochem Int 47(1-2): 71-77.

40. Jayakumar AR, Liu M, Moriyama M, Ramakrishnan R, Forbush B, et al (2008) Na-K-Cl Cotransporter-1 in the mechanism of ammonia-induced astrocyte swelling. J Biol Chem 283(49): 33874-33882.

41. Chu H, Xiang J, Wu P, Su J, Ding H, Tang Y, et al. (2014) The role of aquaporin 4 in apoptosis after intracerebral hemorrhage. J Neuroinflammation 11: 184.

42. Eizayaga F, Scorticati C, Prestifilippo JP, Romay S, Fernandez MA, et al. (2006) Altered blood-brain barrier permeability in rats with prehepatic portal hypertension turns to normal when portal pressure is lowered. World J Gastroenterol 12(9): 1367-1372.

43. Scorticati C, Prestifilippo JP, Eizayaga FX, Castro JL, Romay S, et al. (2004) Hyperammonemia, brain edema and blood-brain barrier alterations in prehepatic portal hypertensive rats and paracetamol intoxication. World J Gastroenterol 10(9): 1321-1324
44. Jayakumar AR, Tong XY, Curtis KM, Ruiz-Cordero R, Abreu MT, et al. (2014) Increased toll-like receptor 4 in cerebral endothelial cells contributes to the astrocyte swelling and brain edema in acute hepatic encephalopathy. J Neurochem 128(6): 890-903.

45. Saliba SW, Ariel RM, Ellen Fortwängler, Johannes Ditrich, Juan CP, et al. (2017) AM404, paracetamol metabolite, prevents prostaglandin synthesis in activated microglia by inhibiting COX activity. Journal of Neuroinflammation 14(1): 246.

46. Roche M, Kelly JP, O’Driscoll M, Finn DP (2008) Augmentation of endogenous cannabinoid tone modulates lipopolysaccharide-induced alterations in circulating cytokine levels in rats. Immunology 125(2): 263271.

47. Costa B, Siniscalco D, Trovato AE, Comelli F, Sotgiu ML, et al. (2006) AM404, an inhibitor of anandamide uptake, prevents pain behaviour and modulates cytokine and apoptotic pathways in a rat model of neuropathic pain. Br J Pharmacol 148(7): 1022-1032.

48. Högestätt ED, Jönsson BA, Ermund A, Andersson DA, Björk $\mathrm{H}$, et al. (2005) Conversion of acetaminophen to the bioactive $\mathrm{N}$-acylphenolamine AM404 via fatty acid amide hydrolase-dependent arachidonic acid conjugation in the nervous system. J Biol Chem 280(36): 3140531412.

49. Sharma C, Long JH, Shah S, Rahman J, Perrett D, et al. (2017) First evidence of the conversion of paracetamol to AM404 in human cerebrospinal fluid. Journal of Pain Research Volume 10: 2703-2709.
Creative Commons Attribution 4.0

International License

For possible submissions Click Here
Submit Article

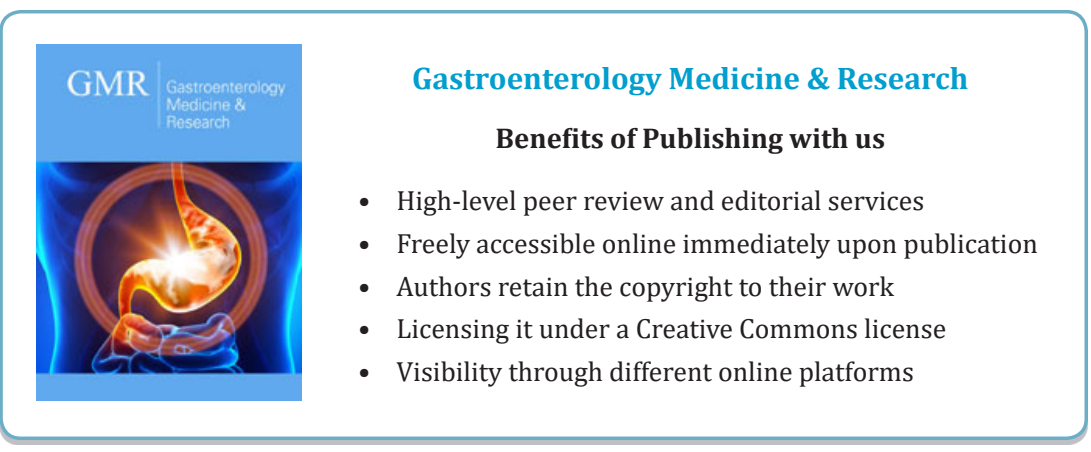

